\title{
BMJ Open Analysis of CTG repeat length variation in the DMPK gene in the general population and the molecular diagnosis of myotonic dystrophy type 1 in Malaysia
}

\author{
Kathlin K Ambrose, ${ }^{1}$ Taufik Ishak, ${ }^{2}$ Lay-Hoong Lian, ${ }^{1}$ Khean-Jin Goh, ${ }^{3}$ \\ Kum-Thong Wong, ${ }^{4}$ Azlina Ahmad-Annuar, ${ }^{5}$ Meow-Keong Thong ${ }^{2}$
}

To cite: Ambrose KK, Ishak T, Lian L-H, et al. Analysis of CTG repeat length variation in the $D M P K$ gene in the general population and the molecular diagnosis of myotonic dystrophy type 1 in Malaysia. BMJ Open 2017;7: e010711. doi:10.1136/ bmjopen-2015-010711

- Prepublication history and additional material is available. To view please visit the journal (http://dx.doi.org/ 10.1136/bmjopen-2015010711).

KKA, TI and M-KT contributed equally to the manuscript.

Received 10 January 2016 Revised 18 August 2016 Accepted 10 October 2016

CrossMark

For numbered affiliations see end of article.

Correspondence to Professor Meow-Keong Thong; thongmk@um.edu.my

\section{ABSTRACT}

Objective: The lack of epidemiological data and molecular diagnostic services in Malaysia has hampered the setting-up of a comprehensive management plan for patients with myotonic dystrophy type 1 (DM1), leading to delayed diagnosis, treatment and support for patients and families. The aim of this study was to estimate the prevalence of DM1 in the 3 major ethnic groups in Malaysia and evaluate the feasibility of a single tube triplet-primed PCR (TP-PCR) method for diagnosis of DM1 in Malaysia.

Design, setting and participants: We used PCR to determine the size of CTG repeats in 377 individuals not known to be affected by DM and 11 DM1 suspected patients, recruited from a tertiary hospital in Kuala Lumpur. TP-PCR was performed on selected samples, followed by Southern blot hybridisation of PCR amplified fragments to confirm and estimate the size of CTG expansion.

Outcome measures: The number of individuals not known to be affected by DM with $(C T G)_{>18}$ was determined according to ethnic group and as a whole population. The $\chi^{2}$ test was performed to compare the distribution of $(\mathrm{CTG})_{>18}$ with 12 other populations. Additionally, the accuracy of TP-PCR in detecting CTG expansion in 11 patients with DM1 was determined by comparing the results with that from Southern blot hybridisation.

Results: Of the 754 chromosomes studied, (CTG) $>18$ frequency of $3.60 \%, 1.57 \%$ and $4.00 \%$ in the Malay, Chinese and Indian subpopulations, respectively, was detected, showing similarities to data from Thai, Taiwanese and Kuwaiti populations. We also successfully detected CTG expansions in 9 patients using the TP-PCR method followed by the estimation of CTG expansion size via Southern blot hybridisation. Conclusions: The results show a low DM1 prevalence in Malaysia with the possibility of underdiagnosis and demonstrates the feasibility of using a clinical and TPPCR-based approach for rapid and cost-effective DM1 diagnosis in developing countries.

\section{Strengths and limitations of this study}

- This is the first myotonic dystrophy type 1 (DM1) epidemiological study on individuals not known to be affected by DM from the three major ethnic groups in Malaysia.

- To date, molecular diagnostic testing for DM1 is not performed in any hospital in Malaysia. This study describes the feasibility of a cost and timeeffective triplet-primed PCR-based method for rapid screening and diagnosis of DM1.

- The genotyping data does not give allele size accurate to one trinucleotide repeat, but rather is a close approximation of the allele size.

- The number of DM1 samples analysed is small as DM1 is a rare disease in Malaysia.

\section{INTRODUCTION}

Myotonic dystrophies (DM) are the most prevalent adult muscular dystrophy worldwide, with an estimated prevalence of 1 in $8000 .{ }^{1}$ They are classified into two main subgroups, DM type 1 (DM1) and type 2 (DM2). These are caused by nucleotide repeat expansions, which are inherited as an autosomal dominant trait, and manifest as clinically heterogeneous diseases. DM1 is due to CTG nucleotide repeats beyond the normal length of $5-49$, in the 3 ' untranslated region of the dystrophia myotonica protein kinase $(D M P K)$ gene, located on chromosome $19 \mathrm{q} 13.3 .^{2}{ }^{3}$ It is a progressive disease and categorised into several subtypes. The congenital form of DM1 is maternally transmitted more frequently, although the disease occurs equally in men and women. ${ }^{1}{ }^{4}$ The general consensus is that the larger the CTG repeat in an individual, the more severe the disease and the earlier the age of onset. It is however, difficult to classify individual DM1 
cases into distinct categories based merely on the size of CTG repeats, as genotype-phenotype correlation often overlap and are not clearly defined. In addition, the repeat sizes have shown variation, both between tissues, and over time in the same tissue. ${ }^{5}{ }^{6}$ This has made disease prognosis difficult. The genetic phenomenon of anticipation can also be observed in the inheritance of the disease, resulting in a more severe form of the disease coupled with an earlier age of onset in subsequent generations. $^{78}$

The prevalence of DM1 varies greatly across populations-it is predominantly seen among the Europeans and Japanese. ${ }^{9}{ }^{10}$ A study also estimated a high disease frequency in the Finnish population. ${ }^{11}$ In Quebec, Canada, a particularly high DM1 prevalence of 1 in 500 has been recorded due to founder effects. ${ }^{12}$ In contrast, it is a rare disease among ethnic sub-Saharan populations, ${ }^{13}$ being almost unheard of with the exception of one case reported in Nigeria. ${ }^{14}$ Two recent cases among African-Americans have also been observed, most likely representing recent population admixture. ${ }^{15}$ In view of this disparity, a study was undertaken to determine the distribution of CTG repeats in African individuals not known to be affected by DM1. It was found that there was a highly significant difference in the distribution of normal CTG alleles larger than 18 between the African population and the European and Japanese populations. ${ }^{13}$ This reiterates a previous theory that CTG alleles between 19 and 30 act as a source of DM1 mutations in subsequent generations. ${ }^{16}$ These findings have formed the basis for the estimation of DM1 incidence within a population. ${ }^{13}$ 17-27

Prior to the establishment of molecular diagnostic tests, DM1 was diagnosed in clinics mainly by observing clinical symptoms and conducting electromyography (EMG) tests, with confirmation by muscle biopsy. ${ }^{28}$ At present, there are several molecular techniques that can be used in making a DM1 diagnosis, rendering little use for the invasive and painful EMG test and muscle biopsy. ${ }^{29}$ However, a single test that is able to detect all ranges of expansion sizes is yet to be established. Laboratories often employ a combination of methods depending on mutation dynamics in the population and available equipment. Conventional PCR can detect the normal range of CTG repeats as well as premutated alleles. Optimised PCR conditions can detect alleles up to $(\mathrm{CTG})_{85}$, whereas those beyond that rely on Southern blot for detection. The triplet-primed PCR (TP-PCR) method was developed to detect the presence of large expanded alleles, thus reducing the number of reflex Southern blot tests. ${ }^{30}$

As a Southeast Asian country, Malaysia has a population consisting mainly of ethnic Malay, Chinese and Indian. There is also a large group of indigenous people belonging to various tribes. While DM1 has not been frequently diagnosed in this country, there is a possibility of underdiagnosis or misdiagnosis due to the lack of awareness about this condition with its diverse presentations.
No study has been performed on the prevalence and incidence of the disease in the predominant ethnic groups, and to the best of our knowledge, diagnostic tests for this disease at the molecular level is not available anywhere in the country. Given the multisystemic and variable phenotypic manifestations in patients, it is therefore important for a simple standard confirmatory diagnostic test to be available, especially when trying to rule out different diagnoses. Here, we report the use of PCR and Southern blot hybridisation methods for the molecular analysis of individuals not known to be affected by DM from the Malay, Chinese and Indian subpopulations, where we studied the length of the CTG alleles in order to predict the prevalence of DM1 in these subpopulations. We also describe the use of a single-tube TP-PCR method for the screening and confirmation of DM1 among Malaysian patients, with the aim of reducing the number of Southern blot tests that need to be performed.

\section{MATERIALS AND METHODS \\ Ethics statement}

The ethics board required that all human participants recruited in the study were briefed on the nature of the study, and provided with an information sheet describing the study. Participants were also assured that their privacy will be protected, and all personal information provided will be kept confidential. Participation in the study was on a voluntary basis, and had no bearing on the quality of care patients received at the hospital.

\section{Sample collection}

Blood samples from 377 randomly selected anonymous blood donors not known to be affected by DM of Malay, Chinese and Indian descent were obtained from the University of Malaya Medical Centre (UMMC) blood bank following oral consent to participate in the study. In addition, 11 patients displaying DM-like symptoms were recruited to this study. Written consent, clinical and familial history were obtained from these patients. The ethnicity of participants was determined to be Malay, Chinese or Indian based on their own admission.

\section{Molecular analysis}

Genomic DNA was extracted from the blood samples using the QIAamp DNA Blood Mini kit according to manufacturer's protocol (QIAGEN, Hilden, Germany).

\section{Conventional PCR}

Analyses of the samples were carried out according to techniques described by Surh et al. ${ }^{31}$ PCR was performed in a final volume of $30 \mu \mathrm{L}$ using the Perkin Elmer GeneAmp PCR system. The forward, 103, 5'-CCA GTT CAC AAA CCG CTC CGA GCG TG-3' and reverse, 96, 5'-GGT GCG TGG AGG ATG GAA CAC GGA C-3' primers were used. The PCR conditions were set as follows: initial denaturation at $96^{\circ} \mathrm{C}$ for $5 \mathrm{~min}$, followed 
by 25 cycles of denaturation, annealing and extension at $96^{\circ} \mathrm{C}, 62^{\circ} \mathrm{C}$ and $72^{\circ} \mathrm{C}$, respectively, for a period of $1 \mathrm{~min}$ for each step. Final extension was performed at $72^{\circ} \mathrm{C}$ for $7 \mathrm{~min}$. The PCR products were sized by gel electrophoresis on $1.5 \%$ agarose gel, at $100 \mathrm{~V}$ for $45 \mathrm{~min}$. The separated products were cut out from the gel, purified using the QIAquick gel extraction kit (QIAGEN, Hilden, Germany) and sent to a service laboratory for sequencing to determine the exact number of CTG repeats.

\section{Triplet-primed PCR}

Analysis of the samples were performed according to techniques described by Singh et al. ${ }^{32}$ Thirteen samples were subjected to TP-PCR analysis-11 individuals with DM1 symptoms and 2 controls not known to be affected by DM. The participants recruited were all adults between the ages of 30 and 60, and one child aged 5. Testing was performed with $100 \mathrm{ng}$ of genomic DNA from blood samples in a reaction volume of $25 \mu \mathrm{L}$. The primers FAM-P1-forward 5'FAM-GGG GCT CGA AGG GTC CTT GT-3' and P2-reverse 5'-GTG CGT GGA GGA TG AAC ACG-3' flanked the CTG repeat region, with the forward primer labelled with FAM (fluorescein) fluorescence. The third primer P3 5'-AGC GGA TAA CAA TTT CAC ACA GGA-3' was designed to bind to the complement of the tail of the fourth primer P4-(CAG) ${ }_{6}$-reverse 5'-AGC GGA TAA CAA TTT CAC ACA GGA CAG CAG CAG CAG CAG CAG-3'. The primer combination was prepared in a ratio of FAM-P1-forward: $\mathrm{P} 4-(\mathrm{CAG})_{6}$-reverse:P3:P2=1.5:1:1.5:1.5, with a final working concentration of 0.6:0.4:0.6:0.6 $\mu \mathrm{M}$. The TP-PCR conditions were set as follows: initial denaturation at $95^{\circ} \mathrm{C}$ for $5 \mathrm{~min}$, followed by 10 cycles each of denaturation $\left(97^{\circ} \mathrm{C}\right)$ for $35 \mathrm{~s}$, annealing $\left(65^{\circ} \mathrm{C}\right)$ for $35 \mathrm{~s}$ and extension $\left(68^{\circ} \mathrm{C}\right)$ for $4 \mathrm{~min}$. Subsequently, 20 cycles of denaturation, annealing and extension were performed, with the extension time increased by $20 \mathrm{~s}$ per cycle to allow for increased yield of PCR product. The products were separated on an ABI PRISM $3130 \times 1$ genetic analyser (Life Tech) and fragment size determined using GeneMarker V.2.6 (Softgenetics, State College, Pennsylvania, USA).

\section{Southern blot hybridisation of PCR amplified fragments}

Southern blot hybridisation of amplified PCR fragments was carried out in samples that only showed single peaks in the electropherograms, which indicated a CTG expansion or homozygosity for a non-expanded allele. The conventional PCR products were transferred overnight from the agarose gel to a positively charged nylon membrane by capillary transfer and fixing of the DNA to the membrane done via the ultraviolet cross-linking method. The membrane was hybridised overnight in a hybridisation buffer with the addition of $20 \mu \mathrm{L}$ alkaline phosphatase-conjugated (CTG) ${ }_{10}$ oligonucleotide at $50^{\circ} \mathrm{C}$. The membrane was then removed and the excess liquid drained off, prior to being washed using preheated wash buffers. Following hybridisation and washing of the membrane, the CDP-Star Detection Reagent was applied and the development of the signals was subsequently carried out by exposing the blot to an autoradiography film. Identification of DM1-positive samples were done by comparing the size of the bands or smears obtained with DNA molecular weight markers. The size of the expansion was determined as the point of highest band intensity on the autoradiograph. To estimate the size of the expansion, the lengths of the $100 \mathrm{bp}$ or $1 \mathrm{~Kb}$ size marker fragments were first converted into logarithmic values. These values were then graphed on the $y$-axis against their migration distance on the $\mathrm{x}$-axis. Using linear regression, a line of best fit was drawn through these points and an equation describing that line was derived. The unknown fragment's migration distance derived from the most intense region of the expanded allele was placed into the equation for the regression line to determine a $\log$ value for the fragment's size. Taking the antilog of this value yielded the unknown fragment's size in base pairs. This value is an estimate of the average repeat number and is dependent on age at sampling.

\section{Statistical analysis}

The frequency of each of the allele present in the 754 chromosomes from the individuals not known to be affected by DM was calculated. Statistical analysis was performed by administering the $\chi^{2}$ test with Yates' correction to compare the distribution of normal large repeats, $(\mathrm{CTG})_{>18}$, with 12 other populations.

\section{RESULTS}

\section{Analysis of DMPK CTG repeat length variation in the} general population

The distribution of $(\mathrm{CTG})_{>18}$ alleles in the Malay, Chinese and Indian subpopulations all point towards a low prevalence of DM1. Figure 1 shows the breakdown of all alleles present in the three subpopulations. (Individual genotyping data is provided in online supplementary files 1-3.) A bimodal allelic distribution was noted-this was in alignment with patterns observed in other populations with low DM1 frequencies. The first peak came from the $(\mathrm{CTG})_{5}$ alleles, which totalled to $33.7 \%$ of all alleles, while the second peak consisted of three alleles, 11-13 that accounted for a majority of $51.1 \%$ of the total alleles. The frequencies for $(\mathrm{CTG})_{>18}$ alleles were $9 / 250=3.60 \%$ (95\% CI $0.0166 \%$ to $0.0672 \%$ ) in the Malay subpopulation, $4 / 254=1.57 \% \quad(95 \%$ CI $0.0043 \%$ to $0.0398 \%$ ) in the Chinese subpopulation, and $10 / 250=4.00 \% \quad(95 \%$ CI $0.0193 \%$ to $0.0723 \%)$ among the Indians. Heterozygosity was measured at $79.9 \%, 77.0 \%$ and $76.2 \%$ in the three subpopulations, respectively, averaging at $77.7 \%$. This result is aligned to those reported in other populations, which ranged from $73.0 \%$ in Europeans ${ }^{17}$ to $92 \%$ in Iranians. ${ }^{26}$

Tables 1 and 2 show the comparison and $\chi^{2}$ analysis of the frequency of $(\mathrm{CTG})_{>18}$ alleles in individuals not known to be affected by DM1 from the three 


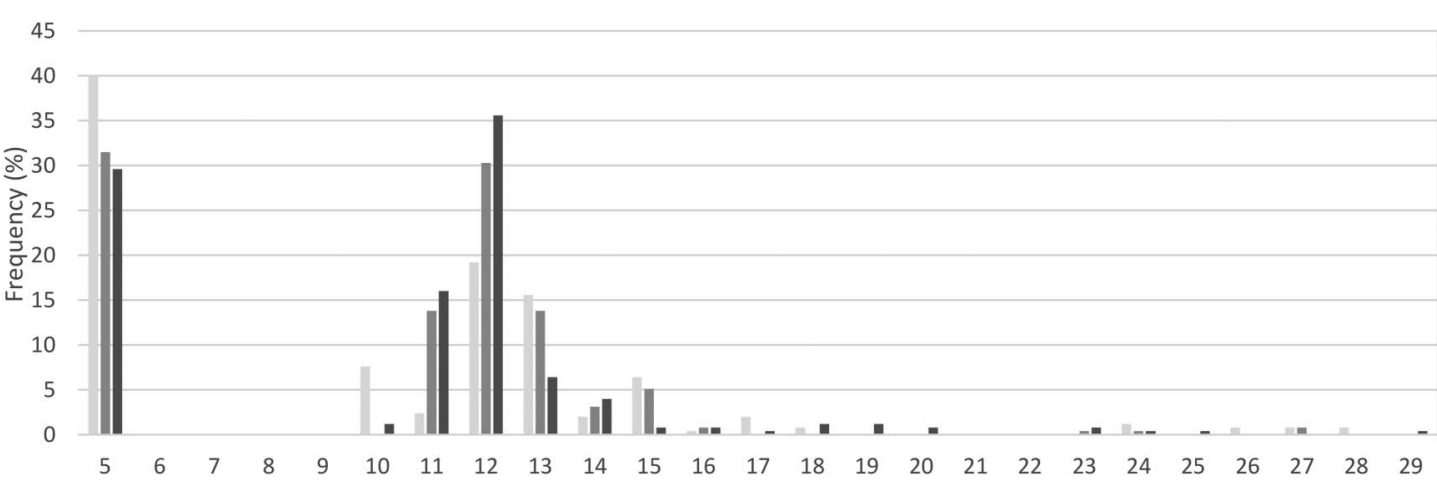

Figure 1 Frequency of CTG repeats in individuals not known to be affected by DM from the Malay, Chinese and Indian subpopulations. The frequency for large normal alleles, (CTG) $>18$ was $9 / 250$ or $3.60 \%$ in the Malays, $4 / 254$ or $1.57 \%$ in the Chinese and $10 / 250$ or $4.00 \%$ in the Indians. A bimodal allelic distribution was observed in the Malaysian population, in alignment with patterns observed in other populations with low DM1 frequency. The most frequently seen allele was $(\mathrm{CTG})_{5}$ in all three subpopulations, whereas $(\mathrm{CTG})_{10-13}$ was the most common allele group. The genotyping data for each individual is provided in the online supplementary files 1-3. DM1, myotonic dystrophy type 1.

Table 1 Comparison and $\chi^{2}$ analysis of the frequency of $(\mathrm{CTG})_{>18}$ alleles in individuals not known to be affected by DM from the Malay, Chinese and Indian subpopulations

\begin{tabular}{lllll}
\hline & $\begin{array}{l}(\mathbf{C T G})_{>18} \text { alleles } \\
\text { /total alleles } \\
\text { analysed }(\%)\end{array}$ & $\begin{array}{l}\text { Comparison of Malay } \\
\text { data with other } \\
\text { populations } \chi^{2} \text { ( } \mathbf{p} \text { value) }\end{array}$ & $\begin{array}{l}\text { Comparison of Chinese } \\
\text { data with other } \\
\text { populations } \chi^{2} \text { ( } \mathbf{p} \text { value) }\end{array}$ & $\begin{array}{l}\text { Comparison of Indian } \\
\text { data with other } \\
\text { populations } \chi^{2}(\mathbf{p} \text { value) }\end{array}$ \\
\hline Malay & $9 / 250(3.60)$ & - & $1.329(0.249)$ & $0.055(0.8151)$ \\
Chinese & $4 / 254(1.57)$ & $1.329(0.249)$ & - & $1.919(0.166)$ \\
Indian & $10 / 250(4.00)$ & $0.055(0.8151)$ & $1.919(0.166)$ & - \\
\hline DM, myotonic dystrophy. & & &
\end{tabular}

Table 2 Comparison and $\chi^{2}$ analysis of the frequency of $(\mathrm{CTG})_{>18}$ alleles in individuals not known to be affected by DM from the 3 Malaysian subpopulations to those in 12 worldwide populations

\begin{tabular}{|c|c|c|c|c|}
\hline Population & $\begin{array}{l}(\mathrm{CTG})_{>18} \text { alleles/ } \\
\text { total alleles } \\
\text { analysed }(\%)\end{array}$ & $\begin{array}{l}\text { Comparison of Malay } \\
\text { data with other } \\
\text { populations } \chi^{2} \text { ( } p \text { value) }\end{array}$ & $\begin{array}{l}\text { Comparison of Chinese } \\
\text { data with other } \\
\text { populations } \chi^{2} \text { ( } p \text { value) }\end{array}$ & $\begin{array}{l}\text { Comparison of Indian } \\
\text { data with other } \\
\text { populations } \chi^{2} \text { ( } p \text { value) }\end{array}$ \\
\hline European $\dagger^{17}$ & 15/130 (11.54) & $7.817\left(0.005^{\star \star}\right)$ & $16.094\left(<0.0001^{\star \star \star}\right)$ & $6.729\left(0.009^{\star \star}\right)$ \\
\hline German $^{18}$ & 22/104 (21.20) & $26.17\left(<0.0001^{\star \star \star}\right)$ & $39.141\left(<0.0001^{\star \star \star}\right)$ & $24.239\left(<0.0001^{\star \star \star}\right)$ \\
\hline Mexican $^{19}$ & $51 / 800(6.38)$ & $2.232(0.135)$ & $8.037\left(0.005^{\star \star}\right)$ & $1.553(0.213)$ \\
\hline Brazilian $^{20}$ & 24/312 (7.69) & 3.497 (0.062) & $9.88\left(0.002^{\star \star}\right)$ & $2.334(0.127)$ \\
\hline Chilean $^{21}$ & $30 / 272(11.00)$ & $9.354\left(0.002^{\star \star}\right)$ & $17.887\left(<0.0001^{\star * \star}\right)$ & $8.131\left(0.004^{\star *}\right)$ \\
\hline Japanese $^{17}$ & $9 / 106(8.50)$ & $2.760(0.097)$ & $8.386\left(0.004^{* *}\right)$ & $2.149(0.143)$ \\
\hline Thai $^{22}$ & $11 / 400(2.75)$ & $0.142(0.706)$ & $0.505(0.477)$ & $0.421(0.516)$ \\
\hline Taiwanese $\mathrm{e}^{23}$ & $7 / 499$ (1.40) & $2.867(0.090)$ & $0.018(0.893)$ & $3.962(0.050)$ \\
\hline Han Chinese 24 & $6 / 600(1.00)$ & $5.463\left(0.019^{\star}\right)$ & $0.134(0.714)$ & $7.052\left(0.008^{\star \star}\right)$ \\
\hline Kuwaiti $^{25}$ & $14 / 370(3.78)$ & $0.010(0.920)$ & $1.894(0.169)$ & $0.006(0.938)$ \\
\hline Iranian 26 & $29 / 400(7.25)$ & $3.090(0.079)$ & $9.292\left(0.002^{* \star}\right)$ & $2.334(0.127)$ \\
\hline South African ${ }^{13}$ & $3 / 420(0.71)$ & $5.869\left(0.015^{\star}\right)$ & $0.457(0.499)$ & $7.249\left(0.007^{\star}\right)$ \\
\hline
\end{tabular}

subpopulations in this study, and in those from 12 worldwide populations, respectively. The $(\mathrm{CTG})_{>18}$ frequency for the Malay, Chinese and Indian subpopulations were significantly different when compared with frequencies in European, German and Chilean populations. All three Malaysian subpopulations showed frequencies similar to Thai ${ }^{22}$ Taiwanese $^{23}$ and Kuwaiti ${ }^{24}$ populations. It is also interesting to note that the Han-Chinese show similarity with the Malaysian Chinese, the population that the majority of Malaysian Chinese trace their ancestry to. This allows for our speculation that the DM1 frequency among Chinese Malaysians is low, similar to that 
observed in the Han-Chinese, ${ }^{24}$ Taiwanese ${ }^{23}$ and South African negroids. ${ }^{13}$

\section{Diagnostic testing for DM1}

Samples from 11 individuals with DM1-like symptoms and two controls not known to be affected by DM were analysed for CTG expansion using TP-PCR followed by confirmation by Southern blot hybridisation of PCR amplified fragments. TP-PCR testing showed single peaks in nine of the samples, and double peaks in the remaining four. The samples with single peaks also showed a clear laddering pattern indicating the presence of CTG expansion (figure 2). Southern blot hybridisation of PCR amplified fragments confirmed the diagnosis of DM1 in the nine samples, with the detection of expanded alleles ranging from a size of 97-690 CTG repeats, as shown in figure 3. Table 3 shows a summary of the characteristics of the disease exhibited by each patient. Figure 4 shows the pedigree diagram and the CTG repeat size of the families and individuals we studied. It is important to note that apart from those diagnosed (dark squares/circles), none of the other family members were examined or tested for DM1. Hence, there is a possibility that there may be family members showing very mild symptoms who have not presented in our clinics, contributing to the apparent under transmission of the disease in the families.

\section{DISCUSSION}

In order to obtain a better understanding of the burden of DM1, we estimated the prevalence of the DM1 using the distribution of CTG alleles larger than 18 in the Malaysian population. The result of $(\mathrm{CTG})_{>18}$ of $3.05 \%$ $(23 / 754)$ was observed in the Malaysian population. By comparing with the results of studies performed in other populations, we predict that DM1 is a rare disease in Malaysia. A larger study is needed to verify these findings, due to the fact that the participants in this study were recruited from a major hospital in the capital city of Malaysia, therefore may not be representative of the whole country. It is likely that DM1 in the local community is underdiagnosed due to a lack of awareness among the public and healthcare professionals. There are also other contributing factors such as social stigma, and reduced access to major hospitals where specialised consultation and testing are available.

Population studies carried out previously have showed evidence for the association of $(\mathrm{CTG})_{>18}$ allele frequency and DM1 prevalence. In European populations, the frequency of DM1 is estimated to be 1 in 8000 which corresponded to $(\mathrm{CTG})_{>18}$ of $\sim 10 \%$. On the other end of the spectrum, DM1 has only been reported in one Southern African Negroid family where the prevalence of $(\mathrm{CTG})_{>18}$ is reported to be $0.7 \%$. In the absence of epidemiological data on real cases of DM1, other populations such as the Brazilian, Mexican, Thai, Taiwanese and Han Chinese report the prevalence of DM1 as either higher or lower than populations with known prevalence.
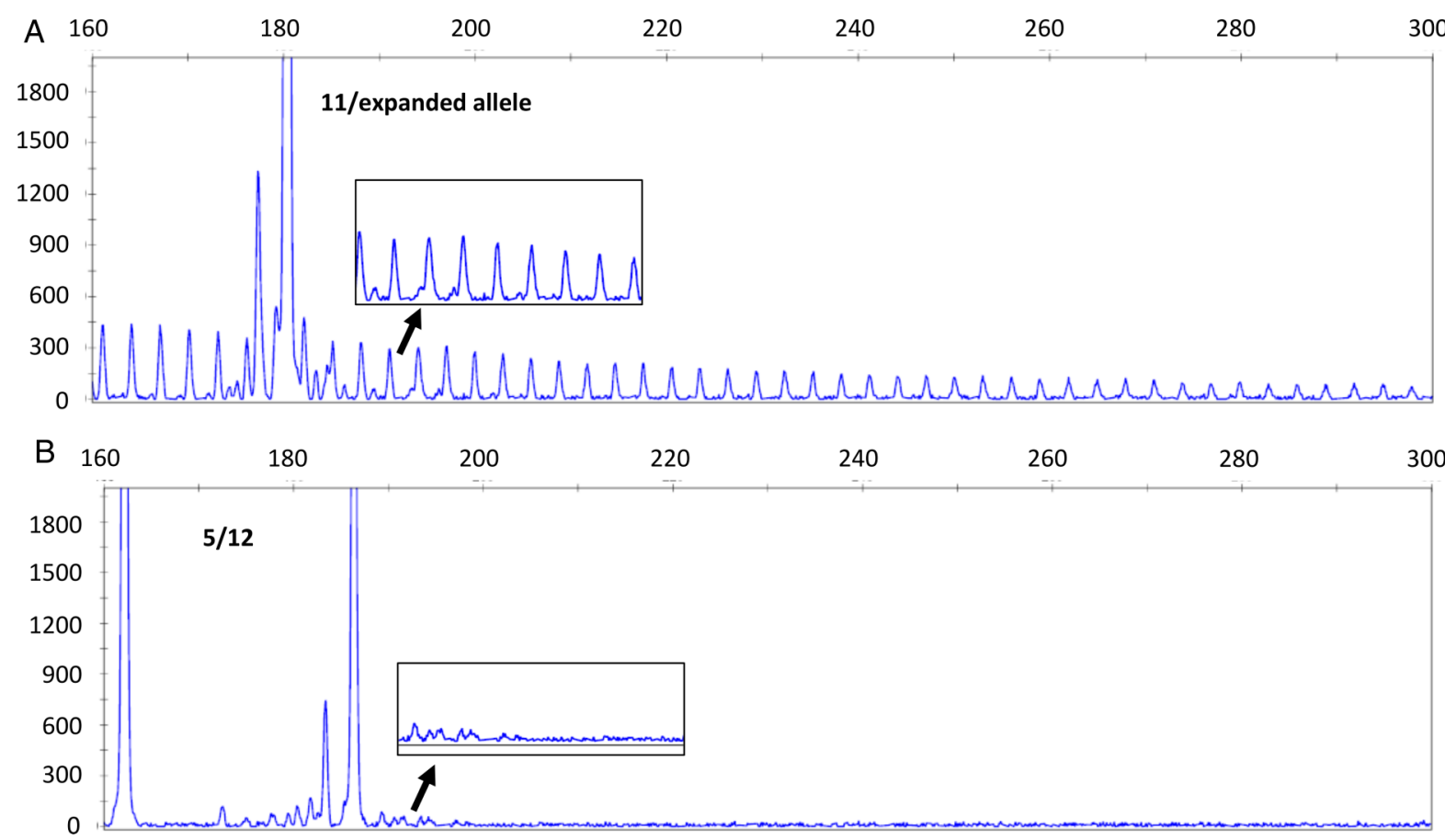

Figure 2 Electropherogram results of TP-PCR. The $x$-axis represents the size in base pairs and the $y$-axis represents the allele peak height. (A) The electropherogram shows a patient sample with DM1 with a single peak corresponding to (CTG) ${ }_{11}$ and a laddering pattern indicating an expanded allele. (B) Two normal heterozygous alleles with sizes 5 and 12 and no laddering pattern observed. DM1, myotonic dystrophy type 1; TP-PCR, triplet-primed PCR. 
Figure 3 Expanded CTG repeats of patients with DM1 Southern blot hybridisation of PCR amplified fragments as seen on an autoradiography film. Expanded alleles in patients ranging from a size of 97 to 690 CTG repeats have been detected. A sample of the bands is shown here, ranging from 270 repeats (1045 bp) to 690 repeats (2305 bp). Normal alleles of four sizes were seen among the patients, 5 (332 bp), 11 (350 bp), 12(353 bp) and 13 (356 bp). Owing to somatic heterogeneity, the expanded alleles usually appear as smears. A $1 \mathrm{~Kb}$ DNA ladder as well as samples from individuals not known to be affected by DM were run alongside patient samples as controls. DM, myotonic dystrophy; DM1, DM type 1.
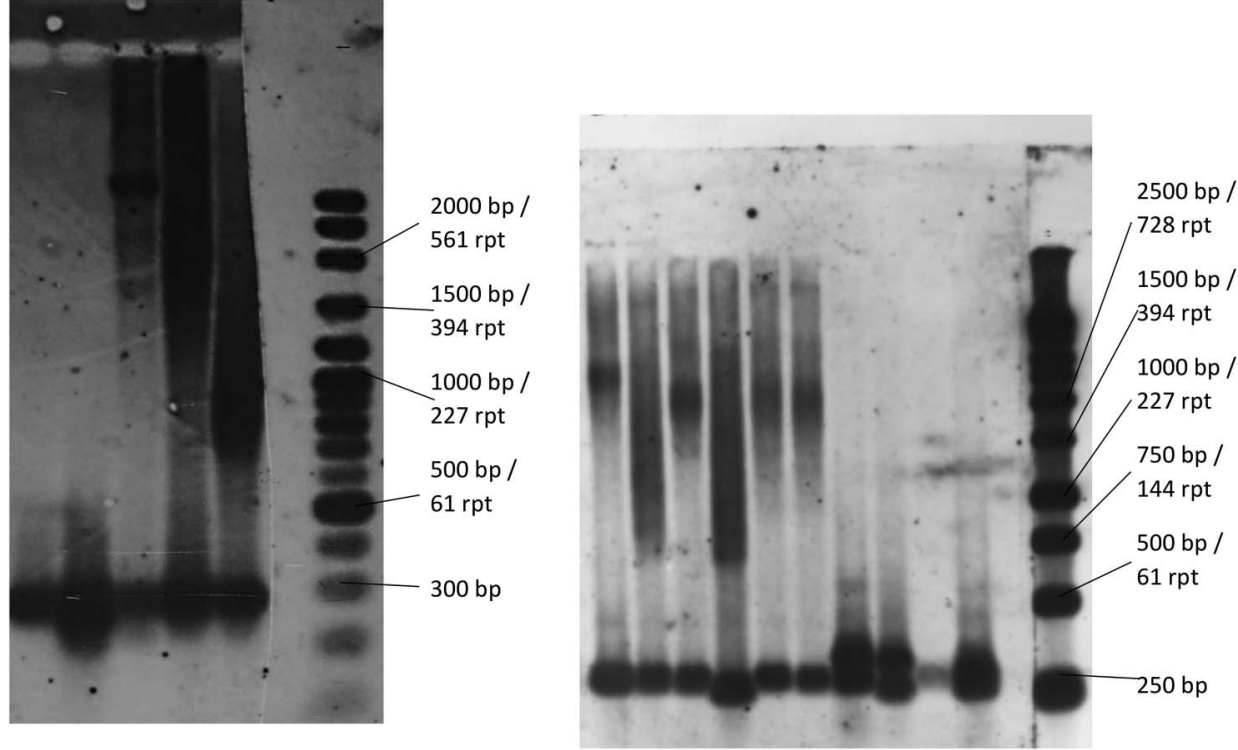

The nine patients identified in this study were diagnosed in the years 2011 and 2012. During these 2 years, the number of patients who were seen at UMMC were 957418 and 964497 , respectively, totalling to 1921915 . Using these figures as guide, we estimate the prevalence of DM1 in Malaysia to be $<1$ in 200000 . This estimate is similar to the low estimates of DM1 prevalence reported in Thai, ${ }^{22}$ Taiwanese ${ }^{23}$ and Kuwaiti ${ }^{25}$ populations, where the authors reported the observed frequencies of alleles $>18$ and correlated them to the prevalence of DM1 in their respective countries. This was also in concordance with the results of our $\chi^{2}$ analysis.

It is interesting to note that the frequency of $(\mathrm{CTG})_{>18}$ was the lowest in the Chinese subpopulation, although they account for the most number of patients with DM1 seen in our hospital (including those not reported here). Indians on the other hand show the highest frequency of $(\mathrm{CTG})_{>18}$ in agreement with the findings that DM1 is highly prevalent in India. ${ }^{33}$ However, the number of Indian patients with DM1 seen in our study was the lowest among the three subpopulations. This may reflect socioeconomic and demographic reasons, as well as misdiagnosis/underdiagnosis of DM1 in the respective subpopulations.

Our study also provides for the first time, data on the $(\mathrm{CTG})_{>18}$ allele frequency in a Malay population. The Malay ethnic group is genetically more similar to the Chinese compared with the Indians. ${ }^{34}$ Comparison of the $(\mathrm{CTG})_{>18}$ distribution of the three ethnic groups, however, shows a closer similarity between the Malays and the Indians $(\mathrm{p}=0.8151)$ compared with the Chinese $(p=0.249)$. It would be interesting to see this same analysis performed on other modern Malay populations in

Table 3 A summary of the disease characteristics exhibited by patients with DM1 in this study

\begin{tabular}{lllllll}
\hline Family & Patient & Gender & Age $^{*}$ & Disease onset† & Phenotype & CTG repeat size \\
\hline 1 & 1 & Male & 54 & Late adult & Classical & 330 \\
& 2 & Male & 30 & Early adult & Classical & 690 \\
2 & 3 & Female & 30 & Early adult & Mild & 350 \\
& 4 & Female & 31 & Early adult & Mild & 97 \\
& 5 & Male & 5 & Birth & Congenital & 596 \\
3 & 6 & Male & 60 & Late adult & Classical & 270 \\
& 7 & Male & 30 & Early adult & Classical & 570 \\
4 & 8 & Male & 44 & Early adult & Classical & 550 \\
5 & 9 & Male & 32 & Early adult & Classical & 520 \\
\hline
\end{tabular}

${ }^{*}$ Age of patient at time of molecular testing.

†Early adulthood: $20-49$ years old; late adulthood: $>50$ years old.

$\ddagger$ Phenotype classification as described by Kamsteeg and colleagues.

DM1, myotonic dystrophy type 1. 

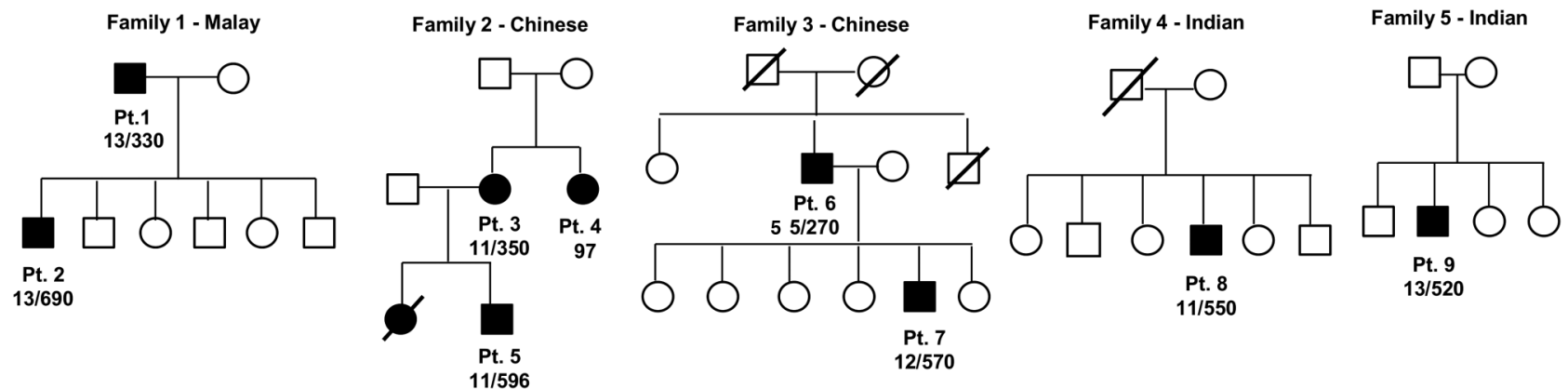

Figure 4 Pedigree diagrams of patients with DM1 studied including the size of their CTG alleles. Members of three families and two individuals had their CTG repeat size analysed. The sizes of the allele pairs for each patient are as stated in the pedigree diagrams. The phenomenon of anticipation was clearly observed in the three families, whereby with the increased CTG expansion in successive generations, a decreasing age of onset is noted. DM1, myotonic dystrophy type 1; Pt, patient.

the region, such as the Singapore Malays and the Indonesians, as well as the aboriginal Malays.

The usage of the single tube TP-PCR allows for the rapid identification of large pathogenic CTG repeats, thus reducing the need for Southern blot-based approaches to detect or exclude the presence of a large expansion. Southern blot may require large amounts of DNA, the use of radioactive materials and is time consuming. In addition, this procedure is also less sensitive and may be difficult to replicate. Hence, any method that reduces the number of Southern blot that needs to be performed, while demonstrating high sensitivity and specificity is advantageous in a clinical setting. However, the TP-PCR test used requires highly specialised equipment, a genetic analyser, which may not yet be widely available and cannot estimate the size of CTG expansions beyond 85 repeats.

Genotype-phenotype correlation studies in patients with DM1 have thus far given conflicting results, with various underlying mechanisms, associations and theories proposed.$^{35-38}$ In particular, in their study on the somatic instability of expanded CTG repeats in DM1, Morales et $a l^{39}$ showed that there was no evidence to indicate that pathogenesis of the disease is constrained to a threshold above which repeat length does not contribute towards age at onset. Additionally, they showed that age at onset is further modified by the level of somatic instability, which is a highly heritable trait. In our study, a disparity in the genotype-phenotype correlation in the Chinese family was seen, whereby patient 3 is largely asymptomatic although she carries 350 repeats. Her disease status was only suspected and diagnosed following the birth of her children who exhibited symptoms. Both her children were congenitally affected, which is consistent with findings in previous studies that showed that the majority of congenital cases were maternally transmitted. Patients 2 and 7 on the other hand paternally inherited their pathogenic alleles, resulting in the classic/adult-onset DM1. The same disease phenotype is seen in patients 8 and 9 . We were not able to determine whether their diseases were inherited, as their parents have never been tested. However, these patients were given genetic counselling and in accordance with ethical principles, have the autonomy of deciding whether or not to disclose their disease status to family members at risk, for future counselling and testing. It was also observed that congenitally affected patient 5 showed a comparable expansion size to those who were classically affected. The only symptoms he has shown, however, are neonatal hypotonia and a mild cognitive dysfunction. The comparable repeat size is most likely due to the younger age of patient 5 compared with the classically affected adults, and suggests that a larger repeat size would be observed, as the patient grows older. Apart from these disease dynamics, there have also been findings of contraction of allele sizes on transmission reported elsewhere. ${ }^{27}{ }^{36}$ All these factors point towards the high complexity of DM1 and illustrate the important need for genetic counselling services to be offered to affected families.

Molecular testing is generally established as the gold standard in diagnosing genetic disorders such as DM1. This is because a molecular test is rapidly able to eliminate differential diagnoses, confirm the DM1 diagnosis and estimate the size of CTG expansion in a patient, thus avoiding the need for invasive procedures such as muscle biopsies. Hilbert et $a t^{40}$ who studied a large cohort of patients with DM enrolled in the US National Registry, explored their diagnostic journeys, which on average took 7 years for a correct DM1 diagnosis to be made. This delay brought about many implications to the patients and their families, ranging from lack of appropriate disease management to missed opportunities for genetic counselling. The situation in many developing countries is much similar or even worse as molecular diagnostic testing for DM1 is not easily available. Potentially, there could be a large number of patients who are undiagnosed/misdiagnosed, as well as those who have been unnecessarily subjected to various investigations for a definitive diagnosis to be made.

The findings from our preliminary study can aid the structuring of a rare disease management framework in Malaysia, using DM1 as a disease model. The data 
presented here add to the scarce literature of DM1 in the Southeast Asian region. The information on CTG repeat lengths of the $D M P K$ gene in individuals not known to be affected by DM, and patents with DM1, together with proper clinical assessment as well as a costeffective molecular approach, carry implications for earlier diagnosis of DM1 and genetic counselling in a low-resource setting.

\section{Author affiliations}

${ }^{1}$ Department of Molecular Medicine, University of Malaya, Kuala Lumpur, Malaysia

${ }^{2}$ Department of Paediatrics, University of Malaya, Kuala Lumpur, Malaysia ${ }^{3}$ Department of Medicine, University of Malaya, Kuala Lumpur, Malaysia ${ }^{4}$ Department of Pathology, University of Malaya, Kuala Lumpur, Malaysia ${ }^{5}$ Department of Biomedical Science, University of Malaya, Kuala Lumpur, Malaysia

Acknowledgements The authors acknowledge the contributions of Juliana Lee who helped in providing genetic counselling and support to the patients and their families. The authors also thank the UMMC blood bank for blood samples of controls, as well as the patients with DM1 for their participation in this study.

Contributors All authors were involved in the conception and design of the work as well as the final approval of the submitted manuscript. KKA and $\mathrm{TI}$ were involved in the acquisition and analysis of data and KKA, TI and M-KT were involved in the drafting of the manuscript. LHL, KJG, KTW, AA-A and M-KT contributed to the critical evaluation of the manuscript.

Funding This study was supported by grants from the Ministry of Higher Education, Malaysia (Fundamental Research Grant Scheme; grant number: FG029/2010A), University of Malaya (Postgraduate Research Fund; grant number: PV126/2012A, UM HIR grant number UM.C / 625 / 1 / HIR / MOHE / MED / 27), research grant from the Malaysian Rare Disorders Society and Page Charge Fund from the Institute of Research Management and Monitoring, University of Malaya.

Competing interests None declared.

Ethics approval Ethical approval to conduct this study was obtained from the University of Malaya Medical Centre (UMMC) Ethics Committee (reference numbers 577.17 and 800.6).

Provenance and peer review Not commissioned; externally peer reviewed.

Data sharing statement No additional data are available.

Open Access This is an Open Access article distributed in accordance with the Creative Commons Attribution Non Commercial (CC BY-NC 4.0) license, which permits others to distribute, remix, adapt, build upon this work noncommercially, and license their derivative works on different terms, provided the original work is properly cited and the use is non-commercial. See: http:// creativecommons.org/licenses/by-nc/4.0/

\section{REFERENCES}

1. Harper PS. Myotonic dystrophy. 3rd edn. London: WB Saunders, 2001.

2. Fu YH, Pizzuti A, Fenwick R, et al. An unstable triplet repeat in a gene related to myotonic muscular dystrophy. Science 1992;255:1256-8.

3. Mahadevan M, Tsilfidis C, Sabourin L, et al. Myotonic dystrophy mutation: an unstable CTG repeat in the $3^{\prime}$ untranslated region of the gene. Science 1992;255:1253-5.

4. Turner C, Hilton-Jones D. The myotonic dystrophies: diagnosis and management. J Neurol Neurosurg Psychiatry 2010;81: $358-67$.

5. Wong LJ, Ashizawa T, Monckton DG, et al. Somatic heterogeneity of the CTG repeat in myotonic dystrophy is age and size dependent. Am J Hum Genet 1995;56:114-22.
6. Anvret M, Ahlberg G, Grandell U, et al. Larger expansions of the CTG repeat in muscle compared to lymphocytes from patients with myotonic dystrophy. Hum Mol Genet 1993;2:1397-400.

7. Ashizawa T, Harper PS. Myotonic dystrophies: an overview. In: Genetic instabilities and neurological diseases, 2nd edn. London, UK: Elsevier. 2006:21-36. http://dx.doi.org/10.1016/B978-012369462$1 / 50003-X$

8. Harper PS, Harley HG, Reardon W, et al. Anticipation in myotonic dystrophy: new light on an old problem. Am J Hum Genet 1992;51:10-6.

9. Harper PS. Myotonic dystrophy. 2nd edn. Philadelphia: Saunders, 1989.

10. Osame M, Furusho T. Genetic epidemiology of myotonic dystrophy in Kagoshima and Okinawa districts in Japan. Clin Neurol 1983;23:1067-71.

11. Suominen T, Bachinski LL, Auvinen S, et al. Population frequency of myotonic dystrophy: higher than expected frequency of myotonic dystrophy type 2 (DM2) in Finland. Eur J Hum Genet 2011;19: 776-82.

12. Mathieu J, De Braekeleer M, Prevost C. Genealogical reconstruction of myotonic dystrophy in the Saguenay-Lac-Saint-Jean area (Quebec, Canada). Neurology 1990;56:328-35.

13. Goldman A, Ramsay M, Jenkins T. Absence of myotonic dystrophy in Southern African Negroids is associated with a significantly lower number of CTG trinucleotide repeats. $J$ Med Genet 1994;31:37-40.

14. Krahe R, Eckhart M, Ogunniyi AO, et al. De novo myotonic dystrophy mutation in a Nigerian kindred. Am J Hum Genet 1995;56:1067-74.

15. Acton RT, Rivers CA, Watson B, et al. DMPK-associated myotonic dystrophy and CTG repeats in Alabama African Americans. Clin Genet 2007;72:448-53.

16. Imbert G, Kretz C, Johnson $\mathrm{K}$, et al. Origin of the expansion mutation in myotonic dystrophy. Nat Genet 1993;4:72-6.

17. Davies J, Yamagata H, Shelbourne P, et al. Comparison of the myotonic dystrophy associated CTG repeat in European and Japanese populations. J Med Genet 1992;29:766-9.

18. Deka R, Majumder PP, Shriver MD, et al. Distribution and evolution of CTG repeats at the myotonin protein kinase gene in human populations. Genome Res 1996;6:142-54.

19. Magaña JJ, Cortés-Reynosa P, Escobar-Cedillo R, et al. Distribution of CTG repeats at the DMPK gene in myotonic dystrophy patients and healthy individuals from the Mexican population. Mol Biol Rep 2011;38:1341-6.

20. Freitas SRS, Cabello PH, Moura-Neto RS, et al. Analysis of the $D M P K$ gene CTG repeats in healthy Brazilians. Genet Mol Bio 2007;30:14-16

21. Amenabar $F$, Jorquera $H$, Acuna $M$, et al. CTG repeats at the myotonic protein kinase gene in a healthy Chilean population sample. Acta Neurol Scand 2009;119:321-4.

22. Theerasasawat S, Papsing C, Pulkes T. CTG repeat lengths of the DMPK gene in myotonic dystrophy patients compared to healthy controls in Thailand. J Clin Neurosci 2010;17: 1520-2.

23. Pan H, Lin HM, Ku WY, et al. Haplotype analysis of the myotonic dystrophy type 1 (DM1) locus in Taiwan: implications for low prevalence and founder mutations of Taiwanese myotonic dystrophy type 1. Eur J Hum Genet 2001;9:638-41.

24. Sizhong Z, Hui W, Agen $\mathrm{P}$, et al. Low incidence of myotonic dystrophy in Chinese Hans is associated with a lower number of CTG trinucleotide repeats. Am J Med Genet 2000;96:425-8.

25. Alfadhli S, Elshafey AE, Bastaki L, et al. CTG repeat number at the myotonic dystrophy locus in healthy Kuwaiti individuals. Arch Neurol 2004:61:895-8.

26. Shojasaffar B, Moradin N, Kahrizi K, et al. CTG expansion \& haplotype analysis of DM1 gene in healthy Iranian population. Can $J$ Neurol Sci 2008;35:216-19.

27. Kim SY, Kim JY, Kim GP, et al. Molecular and clinical characteristics of myotonic dystrophy type 1 in Koreans. Korean J Lab Med 2008;28:483-92.

28. Magaña JJ, Cisneros B. Myotonic dystrophy type 1 (DM1): from the genetics to molecular mechanisms. In: Muscular dystrophy. INTECH open access publisher; 2012. http://www.intechopen.com/books/ muscular-dystrophy/myotonic-dystrophy-type-1-dm1-fromthegenetics-to-molecular-mechanisms

29. Kamsteeg EJ, Kress W, Catalli C, et al. Best practise guidelines and recommendations on the molecular diagnosis of myotonic dystrophy types 1 and 2. Eur J Hum Genet 2012;20:1203-8.

30. Warner JP, Barron LH, Goudie D, et al. A general method for the detection of large CAG repeat expansions by fluorescent PCR. J Med Genet 1996;33:1022-6. 
31. Surh LC, Mahadevan M, Korneluk RG. Analysis of trinucleotide repeats in myotonic dystrophy. Curr Prot Hum Genet 2001; Chapter 9:Unit 9.6:9.6.1-9.6.13.

32. Singh S, Zhang A, Dlouhy S, et al. Detection of large expansions in myotonic dystrophy type 1 using triplet primed PCR. Front Genet 2014;5:94.

33. Basu P, Majumder P, Roychoudhury S, et al. Haplotype analysis of genomic polymorphisms in and around the myotonic dystrophy locus in diverse populations of India. Hum Genet 2001;108:310-17.

34. Tan SG. Genetic relationships among sixteen ethnic groups from Malaysia and Southeast Asia. Singapore: World Scientific, 2001:83-92.

35. Kinoshita M, Takahashi R, Hasegawa $\mathrm{T}$, et al. $(\mathrm{CTG})_{\mathrm{n}}$ expansions in various tissues from a myotonic dystrophy patient. Muscle Nerve 1996;19:240-2.
36. Gharehbaghi-Schnell EB, Finsterer J, Korschineck I, et al. Genotype-phenotype correlation in myotonic dystrophy. Clin Genet 1998;53:20-6.

37. Zatz M, Passos-Bueno MR, Cerqueira A, et al. Analysis of the CTG repeat in skeletal muscle of young and adult myotonic dystrophy patients: When does the expansion occur? Hum Mol Genet 1995;4:401-6.

38. Savić D, Rakočvić-Stojanović V, Keckarević D, et al. 250 CTG repeats in DMPK is a threshold for correlation of expansion size and age at onset of juvenile-adult DM1. Hum Mutat 2002;19:131-9.

39. Morales F, Couto JM, Higham CF, et al. Somatic instability of the expanded CTG triplet repeat in myotonic dystrophy type 1 is a heritable quantitative trait and modifier of disease severity. Hum Mol Genet 2012;21:3558-67.

40. Hilbert JE, Ashizawa T, Day JW, et al. Diagnostic odyssey of patients with myotonic dystrophy. J Neurol 2013;260:2497-504. 